\section{Seqüelas Endócrinas da Radioterapia no Tratamento do Câncer na Infância e Adolescência}

\section{RESUMO}

A radioterapia resulta em endocrinopatias, osteoporose, obesidade e seqüelas neurológicas em pacientes tratados por câncer. A deficiência de GH é a complicação mais freqüente no eixo hipotálamo-hipofisário. A freqüência, prazo de surgimento e gravidade da deficiência de $\mathrm{GH}$ dependem da dose recebida durante a irradiação craniana, mas idade à radioterapia e fracionamento da dose também são variáveis importantes. Outras anormalidades do eixo hipotálamo-hipofisário são igualmente dose-dependentes. Baixas doses de irradiação induzem puberdade precoce ou avançada, enquanto altas doses provocam deficiência gonadotrópica. Complicações endócrinas secundárias à irradiação periférica, como distúrbios gonadais ou tireoidianos são descritos. Mesmo com secreção normal de $\mathrm{GH}$, o crescimento pode ser comprometido por lesões ósseas após irradiação corporal total ou crânio-espinhal. Resultados melhores sobre a estatura final têm sido obtidos com reposição de $\mathrm{GH}$ em associação com o tratamento da puberdade precoce ou avançada. O objetivo desta revisão é a abordagem das seqüelas endócrinas tardias da radioterapia. (Arq Bras Endocrinol Metab 2005;49/5:825-832)

Descritores: Radioterapia; Crianças; Crescimento; Seqüelas endócrinas

\begin{abstract}
Endocrine Sequelae After Radiotherapy in Childhood and Adolescence.

Radiotherapy may result in endocrine abnormalities, osteoporosis, obesity and neurological sequelae in patients treated for cancer. In the hypothalamo-pituitary area, GH deficiency is the most frequent complication. The frequency, delay of appearance and severity of GH deficiency depend most on the dose delivered during cranial irradiation but variables as age at treatment and fractionation schedule may play an important role as well. Other hypothalamo-pituitary dysfunctions are also dose-dependent. Low dose cranial irradiation may induce precocious or early puberty, while high doses are related to gonadotropin deficiency. Endocrine complications due to extracranial irradiaton such as gonadal or thyroid abnormalities are described. In spite of normal $\mathrm{GH}$ secretion, linear growth may be impaired by bone lesions secondary to craniospinal or total body irradiation. Results on final height have been optimized by better indicators of GH therapy associated with adequate treatment of early or precocious puberty. The purpose of this review is to explore the late endocrine sequelae of radiotherapy. (Arq Bras Endocrinol Metab 2005;49/5:825-832)
\end{abstract}

Keywords: Radiotherapy; Children; Growth; Endocrine sequelae revisão

\author{
Ana Cláudia Couto-Silva \\ Raja Brauner \\ Luis Fernando Adan
}

Universidade Federal da Bahia (ACC-S, LFA), Salvador, BA; e Universidade Paris $V(R B)$, Paris, França. 
C OMO CONSEQÜÊNCIA DA MAIOR eficácia das modalidades terapêuticas na cura do câncer na infância, uma proporção significativa de pacientes está atingindo a idade adulta, e efeitos colaterais tardios dessas terapias vêm sendo descritos. D entre esses efeitos, com implicações na qualidade de vida, destacam-se: comprometimento do aprendizado e memória, osteoporose, endocrinopatias e obesidade $(1,2)$. A radioterapia empregada no tratamento de neoplasias na infância pode restringir-se ao crânio, estender-se por todo o SNC (Sistema Nervoso Central) ou, em algumas situações, incluir a irradiação corporal total $(\mathrm{TBI}=$ total body irradiation) ou parcial ( $\mathrm{TLI}=$ total lymphoid irradiation). 0 presente artigo visa abordar as seqüelas endócrinas por comprometimento central ou periférico da radioterapia.

\section{EFEITOS DA RADIOTERAPIA NA FUNÇĀO HIPOTÁLAMO-HIPOFISÁRIA}

A insuficiência hipofisária induzida por radioterapia craniana foi descrita pela primeira vez em 1966 (3). Os efeitos deste tratamento sobre a função hipotálamohipofisária são melhor estudados através de pacientes, cuja patologia de base situa-se distante do SNC. Quando o tratamento inclui TBI, adicionam-se os efeitos diretos da radioterapia sobre as cartilagens de crescimento.

O mecanismo pelo qual a irradiação craniana induz anomalias na região hipotálamo-hipofisária é desconhecido, podendo tratar-se de lesão celular e/ ou vascular. 0 sítio da lesão também não está estabelecido. Alguns elementos falam em favor de lesão hipotalâmica, como as respostas normais ao testes do $\mathrm{TRH}$, GnRH e GHRH (4), enquanto que os níveis de tiroxina, esteróides sexuais e a resposta do $\mathrm{GH}$ à estimulação farmacológica estão diminuídos (5). Adicionalmente, os níveis elevados de prolactina sugerem que sua inibição hipotalâmica está suprimida.

As seqüelas endócrinas se manifestam, na maioria das vezes, por distúrbios do crescimento decorrentes das deficiências de GH , de hormônios tireoidianos e, no período puberal, de esteróides sexuais.

\section{Deficiência de GH}

É a mais freqüente das complicações endócrinas após radioterapia craniana. A dose de radiação recebida pela região hipotálamo-hipofisária é a variável que determina a freqüência, o prazo de surgimento e a gravidade da deficiência de GH .
Freqüência quanto maior a dose de irradiação, maior a chance de deficiência de GH , a qual ocorreu em $56 \%$ dos pacientes que receberam $24 \mathrm{G}$ y e em $100 \%$ daqueles cujo tratamento comportou doses superioresa $45 \mathrm{~Gy}$ (6). Prazo de surgimento: quanto maior a dose recebida, mais rapidamente se manifesta a disfunção somatotrópica. Em estudo prospectivo longitudinal, demonstrou-se que o uso de doses entre 31-42 Gy, leva à diminuição da secreção de GH a partir dos 18 meses, instalando-se a deficiência clássica em $60 \%$ dos pacientes irradiados após dois anos (7). N aqueles que receberam doses superiores a $45 \mathrm{~Gy}$, esta deficiência aparece meses após a irradiação e está presente em $100 \%$ dos casos ao final de dois anos (8).

Gravidade: uma vez estabelecida a deficiência de GH , observa-se que a desaceleração estatural é mais acentuada nos que receberam doses maiores que $45 \mathrm{~Gy}$ e inconstante naqueles com doses inferiores a $24 \mathrm{~Gy}(6)$. Fracionamento da dose e idade no momento da radiote rapia: são outros fatores preditivos de deficiência de $\mathrm{GH}$, sendo os mais jovens mais vulneráveis aos efeitos da irradiação (9). D ezoito dos 21 pacientes (86\%) com deficiência de GH após radioterapia craniana (24 Gy) por leucemia haviam sido irradiados antes dos cinco anos de idade (10). Para uma determinada dose, a disfunção é tanto mais freqüente quanto menor for o fracionamento e o espaço de tempo utilizado para o tratamento. Assim, pacientes que receberam TBI estão sob risco de deficiência de $\mathrm{GH}$, visto que, apesar das pequenas doses utilizadas (7-15 Gy), o condicionamento dura apenas de um a três dias (11).

Em pacientes irradiados, existe uma boa concordância entre a secreção de $\mathrm{GH}$ e a velocidade de crescimento. Entretanto, quatro exceções merecem ser mencionadas: a) pacientes que receberam doses baixas de radioterapia podem manter velocidade de crescimento normal a despeito da deficiência de GH , estando sob risco de estirão puberal medíocre; b) pacientes com puberdade precoce e deficiência de GH mantêm velocidade de crescimento normal com risco de baixa estatura final pelo fechamento precoce das epífises ósseas; c) pacientes com lesões das cartilagens de crescimento (após TBI ou irradiação espinhal) podem apresentar desaceleração estatural sem que a secreção de GH esteja necessariamente diminuída; d) pacientes com sobrepeso (craniofaringioma, por exemplo) podem manter velocidade de crescimento normal, apesar da deficiência de GH (12).

\section{Estudo da secreção de GH}

São diversas, as dificuldades diagnósticas: discordância entre testes de estímulo farmacológico e secreção 
espontânea de GH , níveis normais de IGF-1 e I GFBP3 , manutenção de uma velocidade de crescimento adequada a despeito da secreção de GH diminuída, influência de sobrepeso e/ ou retardo puberal sobre 0 pico de GH .

Embora a ocorrência de disfunção neuro-secretória em pacientes irradiados com baixas doses seja descrita (13), a resposta aos testes de estímulo farmacológico, menos penosos para pacientes previamente manipulados, parece fidedigna (7). Os estudos de secreção espontânea ficariam reservados aos pacientes com desaceleração estatural acentuada com picos normais de GH após estímulo farmacológico, e isto após exclusão de outros fatores implicados na baixa estatura, a exemplo da irradiação das cartilagens de crescimento.

O diagnóstico de deficiência de GH também pode ser corroborado pelas dosagens de IGF-1 e IGFBP-3. Apesar de bons marcadores da deficiência de GH idiopática, sobretudo em idade pré-puberal, 0 seu papel nos pacientes irradiados é controverso. Alguns estudos mostram que IGF-1 e IGFBP-3 são comumente normais nesta população $(14,15)$. Seus níveis também são normais ou elevados em pacientes que receberam TBI como condicionamento para transplante de medula óssea, mesmo naqueles com picos de GH insuficientes, sugerindo um mecanismo de resistência ao IGF-1 por irradiação das cartilagens ou, ainda, uma deficiência de GH "menos grave" (16). Por outro lado, estudo com 90 jovens reavaliados ao final do tratamento com rGH demonstrou que o pico de $\mathrm{GH}$ pós-estímulo correlaciona-se com o IGF-1, e ambos inversamente com a dose de irradiação, sugerindo que esta última determina a freqüência de IGF1 diminuído, o qual pode ser utilizado para monitorar a secreção residual de GH (17).

\section{Tratamento com rGH e estatura adulta}

Antes da decisão pelo tratamento com rG H, deve-se considerar a possibilidade, ainda que teórica, de recidiva tumoral ou de uma segunda neoplasia. É conveniente, portanto, aguardar um mínimo de dois anos após a alta oncológica antes de iniciá-lo. O s resultados do tratamento costumam ser menos eficazes do que em casos de deficiência de GH idiopática, piorando de forma significativa em pacientes que receberam TBI ou irradiação crânio-espinhal. Em pacientes após radioterapia craniana por leucemia (não tratados com rGH ), a perda estatural foi de 0,9 DP naqueles com pico de GH normal e de 1,7 DP nos que apresentavam diminuição na secreção de GH (10). N os pacientes com meduloblastoma, estas perdas foram respectivamente de 2,0 e 3,6 DP (18). Mais recentemente, em 56 pacientes tratados com rG H (doses 0,4 - 0,6 UI/ kg/ semana) observou-se estatura final de -1,0 D P após radioterapia craniana e de $-2,0 \mathrm{DP}$ em pacientes que receberam irradiação crânio-espinhal. Este mesmo estudo demonstrou que, no grupo de radioterapia craniana, os pacientes tratados com análogos de GnRH por puberdade precoce ou avançada apresentaram medidas do segmento superior e diferença entre estaturas-alvo e final superiores àqueles com puberdade em idade normal, a despeito de doses similares de irradiação, pico de $\mathrm{GH}$, início, dose e duração do tratamento com rGH (19).

\section{Outras funções hipofisárias}

As demais funções da hipófise anterior são menos freqüentemente acometidas e estão associadas a doses superiores a $24 \mathrm{~Gy}$; diabetes insipidus, por outro lado, não foi descrito após irradiação craniana. Em estudo realizado (4) com pacientes que receberam doses entre 40 e $70 \mathrm{~Gy}$, entre 6 e 65 anos, identificou-se deficiências tireoidiana $(69 \%)$, gonadotrópica (61\%) e corticotrópica (42\%), além de hiperprolactinemia (50\%).

Deficiência de hormônios tireoidianos: nos casos de origem central, o teste do TRH pode ser necessário para confirmação diagnóstica. A reposição com levotiroxina é sempre indicada. D entre os 90 pacientes irradiados com doses entre 18 e $60 \mathrm{G}$ y avaliados ao final do acompanhamento pediátrico, nenhum apresentava sintomas de hipotireoidismo. Entretanto, 33\% deles - com T4 livre diminuído ou resposta anormal ao teste do TRH - eram tratados com levotiroxina. Salienta-se que todos esses haviam recebido doses $\geq$ 30 Gy (17).

Deficiência corticotrópica: a freqüência de disfunção corticotrópica depende da dose de irradiação e do método utilizado para sua avaliação. No estudo de Constine e cols. (4), o cortisol plasmático após estimulação com ACTH ou CRH foi normal na maioria dos pacientes estudados; entretanto, a resposta do 11desoxicortisol à metirapona foi insuficiente em 35\% dos casos, os quais haviam recebido doses superiores a $50 \mathrm{~Gy}$. Verificou-se associação inversa entre os níveis de 11-desoxicortisol e a dose de irradiação recebida. $\mathrm{N}$ a reposição de corticosteróides, deve-se atentar para o risco de doses supra-fisiológicas que repercutam de forma negativa sobre o crescimento linear dos pacientes jovens. 0 tratamento pode restringir-se, portanto, às intercorrências médicas.

Hiperprolactinemia: comumente moderada, é mais 
freqüente nos adultos do que nas crianças. H iperprolactinemia foi identificada em apenas quatro (5\%) de 80 pacientes avaliados ao final da adolescência. O s níveis de prolactina apresentaram correlação positiva com a idade à irradiação e negativa com o tempo transcorrido desde então, mas não com a dose total recebida (17).

Distúrbios puberais: a radioterapia craniana pode alterar as gonadotrofinas hipofisárias de duas maneiras distintas, em função das doses recebidas. A puberdade precoce costuma surgir após irradiação com doses entre 18-24 Gy, enquanto que a insuficiência gonadotrópica é mais freqüente em doses superiores a 35-50 Gy. Existe ainda uma associação direta entre idade à irradiação e idade de surgimento da puberdade (20). Estes autores sugerem a ocorrência de puberdade precoce apenas no sexo feminino, quando as doses de irradiação utilizadas situam-se entre $18 \mathrm{e}$ $24 \mathrm{~Gy}$. Parte dos pacientes irradiados pode apresentar puberdade que se inicia em idade normal, mas de progressão acelerada, resultando em fechamento precoce das epífises ósseas e redução do potencial de crescimento. 0 problema se agrava quando à puberdade precoce/ acelerada associa-se a deficiência de $\mathrm{GH}$. O uso de análogos de GnRH e de GH recombinante (quando indicado) conduz à estatura final adequada $(21,22)$.

A insuficiência gonadotrópica se manifesta por ausência ou parada do desenvolvimento puberal. Em algumas situações, ocorre amenorréia com testes de estímulo com GnRH normais, sugerindo disfunção hipotalâmica. A insuficiência gonadal também pode ser de origem periférica, secundária à irradiação pélvica ou à quimioterapia, como discutido mais adiante. 0 tratamento substitutivo com esteróides sexuais objetiva induzir ganho estatural puberal e desenvolvimento dos caracteres sexuais secundários.

Em alguns pacientes irradiados, à puberdade precoce sucede-se a insuficiência gonadotrópica. Onze entre 90 pacientes avaliados (12\%) no início da vida adulta apresentaram ausência de progressão puberal ou menstruações raras ou amenorréia primária; destes, nove haviam sido tratados com análogos de GnRH (17).

\section{Obesidade e Síndrome Metabólica}

A obesidade - importante pelos estigmas sociais e psicológicos, além de relevante fator de risco cardiovascular - tem sido descrita como efeito colateral tardio relacionado à terapia oncológica, especialmente após tratamento de leucemia linfoblástica aguda (LLA). Entretanto, o papel da radioterapia como fator de risco isolado no desenvolvimento da obesidade ainda é controverso. Sklar e cols. (23), estudando 126 pacientes tratados por LLA, observaram aumento de peso e obesidade apenas naqueles submetidos à irradiação craniana, sendo o ganho ponderal dose-dependente. O utros dois estudos $(24,25)$, contudo, não confirmam estes achados: embora ambos tenham identificado obesidade no sexo feminino (mas não no masculino), esta só foi associada à irradiação craniana no estudo de Gurney e cols. (24).

E $m$ teoria, a obesidade nos pacientes submetidos à irradiação craniana decorre de danos nos centros cerebrais que controlam 0 apetite, 0 comportamento alimentar e a composição corporal (1). Especula-se ainda que o mecanismo de obesidade nesses pacientes seja multifatorial, podendo incluir anormalidades do balanço energético, diminuição da atividade física, alterações hormonais e uso de corticóides $(1,25)$.

Dislipidemia tem sido relatada, sendo a diminuição do H D L-colesterol 0 achado mais consistente (26-28), embora, em sobreviventes de LLA, aumento do colesterol total também tenha sido encontrado. H eikens e cols. (27) observaram elevação do colesterol total e do LDL-colesterol e diminuição do H D L-colesterol, além de aumento da relação cintura-quadril e hipertensão sistólica, em 26 sobreviventes de câncer cerebral. Aumento da espessura da íntima-média do bulbo carotídeo foi outra alteração descrita nesse estudo, sendo considerada como evidência de aterosclerose prematura. Talvensaari e cols. (28) evidenciaram hiperinsulinemia e aumento do risco de desenvolvimento de síndrome metabólica em sobreviventes de câncer na infância. Acredita-se que a deficiência de GH secundária à irradiação craniana seja o principal fator responsável por essas alterações.

No estudo de Adan e cols. (17), 14\% dos pacientes avaliados ao final da adolescência apresentaram IM C > 2 DP, sendo que este correlacionou-se com os níveis séricos de insulina e leptina, mas não com a idade ou dose de irradiação, pico de GH, IGF-1 ou IGFBP-3.

\section{EFEITOS PERIFÉRICOS DA RADIOTERAPIA}

\section{Baixa estatura}

Além do efeito sobre a secreção de $\mathrm{GH}$, a radioterapia crânio-espinhal pode comprometer a estatura através do efeito direto sobre as cartilagens de crescimento. D oses de irradiação espinhal acima de $20 \mathrm{G}$ y podem 
resultar em encurtamento do tronco e conseqüente perda estatural $(7,29,30)$. TBI e TLI também podem levar a lesões ósseas, que seriam responsáveis pela resistência ao IGFI (31). Inicialmente, acreditou-se que a administração fracionada da TBI seria menos nociva às velocidade de crescimento e secreção de GH que a sua administração em dose única (31). Entretanto, após seguimento mais prolongado, demonstrou-se que ambos os esquemas são deletérios (16). D eve-se ainda levar em consideração que o comprometimento da estatura nos casos de transplante de medula óssea (TMO) é de origem multifatorial, sendo que a deficiência de GH , a displasia óssea rádio-induzida, os distúrbios nutricionais, a doença do enxerto versus hospedeiro e o hipotireoidismo podem estar envolvidos.

\section{Distúrbios tireoidianos}

A tireóide é rádio-sensível. A ssim, radioterapia para tratamento da D oença de H odgkin, irradiação crânio-espinhal para tumores cerebrais e irradiação craniana ou TBI para transplante de medula óssea são fatores de risco para desenvolvimento de distúrbios da tireóide $(1,2)$. Hipotireoidsmo: alteração mais freqüente. Pode tratarse de hipotireoidismo franco ou subclínico e costuma ocorrer nos cinco primeiros anos após o tratamento radioterápico, embora casos novos continuem a aparecer muitos anos após a terapia. Q uanto maior a dose de irradiação cervical, maior o risco de desenvolvimento de hipotireoidismo (1,32-34). Assim, 30\% dos pacientes que receberam entre $35-45 \mathrm{G}$ y e $50 \%$ daqueles irradiados com doses superiores a $45 \mathrm{G}$ y terão hipotireoidismo vinte anos após o tratamento (32).

Hipertireoidismo: complicação rara da radioterapia, na maioria dos casos tem quadro clínico idêntico ao da D oença de Graves $(1,32)$. Tireoidite aguda, ocorrendo durante ou logo após irradiação cervical, também já foi descrita, sendo habitualmente transitória e subclínica.

Nódulos e Câncer da Tireóide: nódulos tireoidianos benignos e malignos ocorrem com freqüência aumentada após radioterapia cervical. A incidência de nódulos nos sobreviventes de Doença de $\mathrm{H}$ odgkin varia entre $2-65 \%$, na dependência do acompanhamento e dos métodos empregados na sua detecção. 0 câncer de tireóide parece ser mais freqüente após doses mais elevadas de irradiação e o período de latência para 0 seu desenvolvimento varia de 5 a 26 anos. 0 sexo masculino parece ter risco absoluto maior que o feminino (32). 0 tipo histológico mais comum de câncer rádio-induzido é o carcinoma papilífero, mas tumores foliculares também podem ocorrer (1).

\section{Distúrbios gonadais}

Radioterapia extra-craniana afetando as gônadas é administrada nos casos de envolvimento testicular por LLA, como parte da TBI e como irradiação abdominal nos pacientes com doença de $\mathrm{H}$ odgkin e tumor de Wilms. O s efeitos da radioterapia na função gonadal variam em função da idade e sexo do paciente e da dose de irradiação utilizada (35).

Comprometimento ovariano: o ovário é menos vulnerável à radioterapia que o testículo. 0 s efeitos da irradiação sobre o tecido ovariano são dose e idadedependentes. Pacientes que recebem radioterapia entre nascimento e 13 anos estão menos sujeitas ao risco de falência ovariana prematura (FOP) do que aquelas tratadas após esta idade (1). D oses ovarianas menores que 4 Gy não costumam levar à disfunção ovariana permanente, enquanto que doses maiores que 20 Gy e TBI levam à falência ovariana na maioria das crianças e adolescentes. Q uase $100 \%$ das pacientes que têm mais de 10 anos no momento da TBI e $50 \%$ daquelas abaixo desta faixa etária irão desenvolver FOP. Algumas pacientes deste grupo podem recuperar a função gonadal com restauração dos níveis de $\mathrm{LH}$, FSH e estradiol a valores normais e menstruação espontânea (36).

Comprometimento testicular: pequenas doses de irradiação são capazes de provocar acentuado comprometimento da função testicular. As células de Leydig são mais resistentes à radioterapia que 0 epitélio germinativo, sendo a insuficiência androgênica incomum (36). Entretanto, a falência compensada (níveis elevados de LH e normais de testosterona) não é infreqüente. D oses acima de $20 \mathrm{G}$ y são requeridas para 0 dano das células de Leydig, as quais parecem ser mais vulneráveis no testículo pré-púbere do que no adolescente (37). I rradiação gonadal pode causar dano das células germinativas em ambos os sexos, sendo 0 tecido testicular mais sensível que o ovariano. Retorno das gonadotrofinas a valores normais não foi descrito nos pacientes do sexo masculino (36).

\section{Distúrbios ósteo-metabólicos}

O steopenia é um importante efeito tardio do tratamento de câncer na infância, sendo sua etiologia multifatorial. Alguns estudos têm demonstrado associação entre radioterapia e osteopenia, que pode ocorrer fora do campo de irradiação $(38,39)$. Especula-se que a diminuição da densidade mineral óssea (DMO) seja decorrente da produção de citocinas e de fatores que afetam a remodelação óssea e a atividade osteoblástica e osteoclástica pelo próprio tumor ou pela irradiação. O utra hipótese seria a perda aguda da DMO pela irradiação per se (39). Q uimioterapia, incluindo o uso 
de corticosteróides e metotrexate, deficiências de hormônio do crescimento, de esteróides sexuais e de hormônio tireoidiano, além de deficiência nutricional, são outros fatores que podem estar envolvidos na patogênese da osteopenia (40).

\section{Distúrbios das paratireóides}

Alguns estudos sugerem aumento de risco de desenvolvimento de hiperparatireoidismo em pacientes que receberam irradiação cervical, sendo longo (25-47 anos) o período de latência entre a radioterapia e 0 desenvolvimento da disfunção. Como muitos desses pacientes permanecem assintomáticos, é difícil assegurar que a irradiação cervical é um fator de risco para o desenvolvimento de hiperparatireoidismo (41).

\section{ASPECTOS PECULIARES DO CRESCIMENTO NAS DIVERSAS PATOLOGIAS}

\section{Meduloblastoma da fossa posterior}

0 tratamento de pacientes com meduloblasto ma comporta uma radioterapia crânio-espinhal que resulta em desaceleração estatural acentuada por comprometimento do segmento superior (tronco curto). 0 acometimento estatural manifesta-se no primeiro ano após a irradiação, mesmo antes da deficiência de GH estabelecer-se. $\mathrm{O}$ s resultados do tratamento com $\mathrm{rGH}$ são comumente medíocres em comparação aos observados em pacientes que receberam apenas radioterapia craniana. A irradiação caudal por si só é responsável por uma perda de um a dois desvios-padrão, equivalente a 5 a $10 \mathrm{~cm} \mathrm{(42).}$

\section{Leucemias}

O tratamento de pacientes com leucemia comporta quimioterapia isolada ou associada à radioterapia craniana e/ ou transplante de medula óssea. As doses de irradiação utilizadas foram reduzidas ao longo de alguns anos, em média de 24 para $18 \mathrm{~Gy}$, com 0 objetivo de diminuir os efeitos colaterais. Apesar disso, a freqüência de deficiência de $\mathrm{GH}$ foi de $63 \% \mathrm{em}$ pacientes com leucemia que receberam $18 \mathrm{~Gy}(43)$, similar aos pacientes que receberam $24 \mathrm{~Gy}$ (10).

\section{Gliomas de vias ópticas}

A puberdade precoce é o distúrbio endócrino mais freqüentemente observado antes da radioterapia (8). Apesar da proximidade com a região hipotálamohipofisária, os gliomas de vias ópticas não cursam por si só com deficiência de $\mathrm{GH}$, a qual aparece menos de dois anos após a irradiação com altas doses (45-55 Gy). N estes pacientes, não é raro que à puberdade
Quadro 1. Indicações para avaliação endócrina de pacientes pós-radioterapia para tratamento de câncer na infância ou adolescência.

\section{Antes de dois anos}

1. Na presença de sinais de puberdade avançada/acelerada; 2. Irradiação da tireóide;

3. Irradiação de ovários e testículos em idade puberal.

\section{Após dois anos}

1. Doses de irradiação > $30 \mathrm{~Gy}$ (mesmo com velocidade de crescimento normal);

2. Diminuição da velocidade de crescimento independente da dose de irradiação;

3. Todos os pacientes em idade puberal, mesmo na ausência de queixas.

precoce suceda-se a deficiência gonadotrópica.

\section{Transplante de medula óssea (TMO)}

O condicionamento para o TM O inclui quimioterapia associada, em alguns pacientes, à TBI ou TLI. A este tipo de irradiação segue-se desaceleração da velocidade de crescimento de difícil avaliação, podendo decorrer de deficiência de $\mathrm{GH}$, acometimento de cartilagens de crescimento, ausência de puberdade por lesão gonadal, além de complicações pulmonares, hepáticas ou renais por GVHD (graft-persus-host disease, doença do enxerto versus hospedeiro) que requerem corticoterapia prolongada. Os resultados do tratamento da deficiência de GH após condicionamento para TM O ainda são inconclusivos, variando de uma modesta melhora (44) até o completo restabelecimento do crescimento aos padrões normais (2).

\section{INDICAÇŌES PARA AVALIAÇĀO ENDÓCRINA PÓS-RADIOTERAPIA}

N osso protocolo atual propõe avaliação endócrina sistemática apenas dois anos após a alta oncológica (quadro 1). Este prazo foi definido com vistas a minimizar as seqüelas orgânicas e psicológicas do tratamento do câncer e leva em conta o maior risco de recidiva nos primeiros anos pós-tratamento. Exceções são feitas em casos de irradiação gonadal durante a puberdade ou diretamente sobre a tireóide.

\section{REFERÊNCIAS}

1. Gleeson HK, Shalet SM. Endocrine complications of neoplastic diseases in children and adolescents. Curr Opin Pediatr 2001;13:346-51. 
2. Sanders JE. Endocrine complications of high-dose therapy with stem cell transplantation. Pediatr Transplantation 2004;8(suppl. 5):39-50.

3. Tan BC, Kunaratnam N. Hypopituitary dwarfism following radiotherapy for nasopharyngeal carcinoma. Clin Radiol 1966; 17:302-4.

4. Constine LS, Woolf PD, Cann D, Mick G, McCormick K, Raubertas RF, et al. Hypothalamic-pituitary dysfunction after radiation for brain tumors. N Eng J Med 1993;328: 87-94.

5. Shalet SM. Radiation and pituitary dysfunction. $\mathbf{N}$ Eng $\mathbf{J}$ Med 1993;328:131-3.

6. Rappaport R, Brauner R. Growth and endocrine disorders secondary to cranial irradiation. Pediatr Res 1989;25:561-7.

7. Brauner R, Rappaport R, Prevot C, Czernichow $P$, Zucker $J M$, Bataini $P$, et al. A prospective study of the development of $\mathrm{GH}$ deficiency in children given cranial irradiation and its relation to statural growth. J Clin Endocrinol Metab 1989;68:346-5.

8. Brauner R, Malandry F, Rappaport R, Zucker ZM, Kalifa C, Pierre-Kahn A, et al. Growth and endocrine disorders in optic glioma. Eur J Pediatr 1990;149:825-8.

9. Brauner R, Czernichow P, Rappaport R. Greater susceptibility to hypothalamo-pituitary irradiation in younger children with acute lymphoblastic leukemia. J Pediatr 1986;108:332.

10. Adan L, Souberbielle JC, Blanche S, Leverger G, Schaison G, Brauner R. Adult height after cranial irradiation with $24 \mathrm{~Gy}$ : factors and markers of height loss. Acta Pediatrica 1996;85:1096-101.

11. Brauner R, Adan L, Souberbielle JC. Surveillance endocrinologique après irradiation du crâne dans l'enfance. Médecine Thérapeutique Endocrinologie 2001;3:2247.

12. Pinto $G$, Brussières $L$, Recasens $C$, Souberbielle JC, Zerah $M$, Brauner R. Hormonal factors influencing weight growth pattern in craniopharyngioma. Horm Res 2000;53:163-9.

13. Darzy KH, Shalet SM. Radiation-induced growth hormone deficiency. Horm Res 2003;59(suppl 1):1-11.

14. Achermann JC, Hindmarsh PC, Brook CGD. The relationship between the growth hormone and insulinlike growth factor axis in long-term survivors of childhood brain tumors. Clin Endocrinol 1998;49:63945.

15. Sklar C. Efficacy of insulin-like growth factor binding protein 3 in predicting the growth hormone response to provocative testing in children treated with cranial irradiation. Acta Endocrinologica 1993;129:51 1-5.

16. Brauner R, Adan L, Souberbielle JC, Esperou A, Michon $\mathrm{J}$, Devergie A, et al. Contribution of growth hormone deficiency to the growth failure that follows bone marrow transplantation. J Pediatr 1997;130:785-92.

17. Adan L, Trivin C, Sainte-Rose C, Zucker JM, Hartmann O, Brauner R. GH deficiency caused by cranial irradiation during childhood: factors and markers in young adults. J Clin Endocrinol Metab 2001;86:5245-51.
18. Sulmont V, Brauner R, Fontoura M, Rappaport R. Response to growth hormone treatment and final height after cranial or craniospinal irradiation. Acta Pediatr Scand 1990;79:542-9.

19. Adan L, Sainte-Rose C, Souberbielle JC, Zucker JM, Kalifa C, Brauner R. Adult height after growth hormone (GH) treatment for $\mathrm{GH}$ deficiency due to cranial irradiation. Med Ped Oncol 2000;34:14-9.

20. Shalet SM, Brennan B. Puberty in children with cancer. Horm Res 2002;57(suppl. 2):39-42.

21. Adan L, Souberbielle JC, Zucker JM, Pierre-Kahn A, Kalifa C, Brauner R. Adult height in 24 patients treated for growth hormone deficiency and early puberty. J Clin Endocrinol Metab 1997;89:229-33.

22. Gleeson HK, Stoeter A, Ogilvy-Stuart AL, Gattamaneni HR, Brennan BM, Shalet SM. Improvements in final height over 25 years in growth hormone (GH)-deficient childhood survivors of brain tumors receiving $\mathrm{GH}$ replacement. J Clin Endocrinol Metab 2003;88:3682-9.

23. Sklar C, Mertens A, Walter A, Mitchell D, Nesbit M, O'Leary $M$, et al. Changes in body mass index and prevalence of overweight in survivors of childhood acute lymphoblastic leukemia: role of cranial irradiation. Med Pediatr Oncol 2000;35:91-5.

24. Gurney JG, Ness KK, Stovall M, Wolden S, Punkyo JA, Neglia JP, at al. Final height and body mass index among adult survivors of childhood brain cancer: childhood cancer survivor study. J Clin Endocrinol Metab 2003;88:4731-9.

25. Warner JT, Evans WD, Webb DKH, Gregory JW. Body composition of long-term survivors of acute lymphoblastic leukemia. Med Pediatr Oncol 2002;38:165-72.

26. Murray RD, Darzy KD, Gleeson HK, Shalet SM. Growth hormone deficient survivors of childhood cancer: growth hormone replacement during adult life. J Clin Endocrinol Metab 2002;87:129-32.

27. Heinkens J, Ubbink MC, van der Pal HP, Baker PJ, Fliers $E$, Smilde TJ, et al. Long-term survivors of childhood brain cancer have an increased risk of cardiovascular disease. Cancer 2000;88:21116-21.

28. Talvensaari KK, Lanning M, Tapanainen P, Knip M. Long term survivors of childhood cancer have increased risk of manifesting the metabolic syndrome. J Clin Endocrinol Metab 1996;81:3051-5.

29. Darendeliler F, Livesey EA, Hindmarsh PC, Brook CG. Growth and growth hormone secretion in children following treatment of brain tumours with radiotherapy. Acta Paediatr Scand 1990;79:950-6.

30. Shalet SM, Gibson B, Swindell R, Pearson D. Effect of spinal irradiation on growth. Arch Dis Child 1987,62:4614.

31. Brauner R, Fontoura M, Zucker JM, Devergie A, Souberbielle JC, Prevot-Saucet C, et al. Growth and growth hormone secretion after bone marrow transplantation. Arch Dis Child 1993;68:458-63.

32. Sklar C, Whitton J, Mertens A, Stovall M, Green D, Marina $\mathrm{N}$, et al. Abnormalities of the thyroid in survivors of Hodgkin's disease: data from the childhood cancer 
study survivor. J Clin Endocrinol Metab 2000;85:322732.

33. Schmiegelow M, Ferdlt-Rasmussen U, Rasmussen AK, Poulsen HS, Müller J. A population-based study of thyroid function after radiotherapy and chemotherapy for a childhood brain tumor. J Clin Endocrinol Metab 2003;88: 136-40.

34. Gurney JG, Kadan-Lottick NS, Packer RJ, Neglia JP, Sklar $\mathrm{CA}$, Punyko JA, et al. Endocrine and cardiovascular late effects among adults survivors of childhood brain tumors. Cancer 2003:97:663-73.

35. Müller J. Impact of cancer therapy on the reproductive axis. Horm Res 2003;59(suppl. 1):12-20.

36. Couto-Silva AC, Trivin C, Thibaud E, Esperou H, Michon J, Brauner R. Factors affecting gonadal function after bone marrow transplantation during childhood. Bone Marrow Transplant 2001;28:67-75.

37. Shalet SM, Tsatsoulis A, Whitehead E, Read G. Vulnerability of the human Leydig cell to radiation damage is dependent upon age. J Endocrinol 1989;120:161-5.

38. Gilsanz V, Carlson ME, Roe TF, Ortega JA. Osteoporosis after cranial irradiation for acute lymphoblastic leukemia. J Pediatr 1990; 117:238-44.
39. Krishnamoorthy P, Freeman C, Bernstein M, Lawrence S, Rodd C. Osteopenia in children who have undergone posterior fossa or craniospinal irradiation for brain tumors. Arch Pediatr Adolesc Med 2004; 158:491-6.

40. Vassilopoulou-Sellin R, Brosnan P, Delpassand A, Zietz H, Klein MJ, Jaffe N. Osteopenia in young adult survivors of childhood cancer. Med Pediatr Oncol 1999;32:272-8.

41. Gleeson HK, Darzy K, Shalet SM. Late endocrine, metabolic and skeletal sequelae following treatment of childhood cancer. Best Prac Res Clin Endocrinol Metab 2002; 16:335-48.

42. Brauner R. Retentissement hypothalamo-hypophysaire des traitements des cancers. Annales d' Endocrinologie (Paris) 1995;56:127-31.

43. Melin AE, Adan L, Leverger $G$, Souberbielle JC, Schaison $G$, Brauner R. Growth hormone secretion, puberty and adult height after cranial irradiation with 18 grays for leukemia. Eur J Pediatr 1998;157:703-7.

44. Couto-Silva AC, Trivin C, Esperou H, Michon J, Fischer A, Brauner R. Changes in height, weight and plasma leptin after bone marrow transplantation. Bone Marrow Transplant 2000;26:1205-10.

\section{Endereço para correspondência:}

\section{Luís Fernando Adan}

Rua Altino Serbeto de Barros 295, apto. 803

41810-570 Salvador, BA 